\title{
Growth Optimization of Averhoa carambola through in vitro Culture Supplemented with Selected Plant Growth Regulators)
}

\author{
Husna Idris, Nor Azlina Hasbullah, Nurul Shifa Radzuan, Fadhlul Khaliq Bin Ab Patah and Mohammad \\ Mohd Lassim
}

\begin{abstract}
Averhoa carambola is one on the important fruit crop in most countries especially in Malaysia. Research on Averhoa carambola were undertaken to investigate the morphogenesis of the plant through tissue culture techniques. The optimal conditions that are required by plant for the purpose of regeneration and also propagation were identified. Various kinds of hormone combinations with different concentrations used for this purpose. Among the hormone Benzylaminopurine (BAP) and Napthalena-Acetic-Acid (NAA) were used to initiate the cultures. In this experiment I have used 16 treatments from different concentration and combination. The optimum medium for shoot regeneration was treatment $14(0.5 \mathrm{mg} / \mathrm{L}$ BAP and mg/L NAA) with $15.4 \pm 7.5$ number of shoot per explants. Meanwhile, for callus induction Meanwhile, for callus induction, stem was observed to be the most responsive explants wthen cultured on MS medium 6 (0.5 mg/L BAP and $0.5 \mathrm{mg} / \mathrm{L}$ NAA) with $100 \%$ callus. This study was conducted at the Crop Science Laboratory, Department of Agricultural Science, in Campus Sultan Abdul Jalil Shah, Sultan Idris Education University (UPSI), Tanjung Malim, Malaysia.
\end{abstract}

Index Terms - Averhoa carambola, explants, growth regulators, MS medium, tissue culture, micropropagation, in vitro culture.

\section{INTRODUCTION}

During normal activities, various processes inside the human body produce reactive oxygen species (ROS) which often cause cell death and are involved in other degenerative processes associated with ageing [4]. As a natural defense system, our body is protected against these free radicals by antioxidant molecules and antioxidant enzymes (Gao et al, 2011). Fruits and vegetables are good plant sources that provide dietary antioxidant to human thealth [2].

Husna Idris is with Department of Agriculture Science, Faculty of Technical and Vocational (FTV), Universiti Pendidikan Sultan Idris (UPSI), Tanjong Malim, Perak,

Dr Nor Azlina Hasbullah is with Department of Agriculture Science, Faculty of Technical and Vocational (FTV), Universiti Pendidikan Sultan Idris (UPSI), Tanjong Malim,Perak, Malaysia

Nurul Shifa Radzuan is with Department of Agriculture Science, Faculty of Technical and Vocational (FTV), Universiti Pendidikan Sultan Idris (UPSI), Tanjong Malim, Perak, Malaysia..

Fadhlul Khaliq Bin Ab Patah is with Department of Agriculture Science, FTV, Sultan Idris Education University (UPSI), 35900 Tanjung Malim, Perak, Malaysia.

Prof. Dr. Mohammad Mohd Lassim is with Faculty of Sustainable Agriculture (Sandakan Campus), Universiti Malaysia Sabah (UMS), 90000, Sandakan, Sabah, Malaysia.
This dietary antioxidant is complemented to antioxidants that naturally produced in human body. It can enhance the potency of antioxidant in scavenging oxidative stress in human body and thereby reduce the risk of having some chronic diseases [1]. Furthermore, the advantages of natural antioxidants in foods are high consumer acceptance due to these health issues [2].

Star fruit (Averhoa carambola) is one of the under utilised tropical fruits that belong to Oxalidaceaa family. It is popular in Southeast Asia countries and has been cultivated in several countries for its edible fruits. Star fruit is sweet in taste and usually consumed fresh or made into juice. Several studies revealed that star fruit containing as high antioxidant level as guava, papaya and banana [3]. With substantial promotions and research evidences, this under utilised fruit could play an important role as an alternative food source to the world and in improving human health. However, only a few publications were reported on the functional potentials of star fruit. Therefore, the current study was undertaken to investigate the total antioxidant contents and capacities of star fruit.

\section{MATERIAL AND METHOD}

\section{A. Seed Germination and Explant Preparation}

The starfruit seed were germinated in sterile culture medium. First, seeds of Averhoa carambola . were separated from the mature ripe fruits by removing the juicy pulp. These seeds were peeled off from coating then drops 4 drops of Tween 20 for 1 hour and then thoroughly wasted under running tap water for 30 minutes to remove all traces of detergent. After that, it has agititated with $70 \%, 50 \%, 30 \%$ and $10 \%$ of alcohol for 1 hour. Seeds were then treated with $70 \%$ alcohol for 1-2 minutes and finally rinsed thrice with autoclaved water. The seeds were then inoculated on basal Murashige and Skoog medium. Let the seedling to grow for a month to ensure the fully develop of leave, shoot and root.

\section{B. Media and Culture Condition}

Seeds in basic medium solution were supplied with $100 \mathrm{ml}$ of macronutrient, $10 \mathrm{ml}$ of micronutrient, $10 \mathrm{ml}$ of vitamin, $10 \mathrm{ml}$ of iron, $30 \mathrm{~g}$ of sucrose and solidified by $8 \mathrm{~g}$ of technical agar in $1000 \mathrm{ml}$ of distilled water. The explants were incubated in constant condition, 16 hours of artificial light and 8 hours of darkness at $23 \pm 3{ }^{\circ} \mathrm{C}$. After 8 to 12 weeks the explants were 
ready to test on several combinations of hormones.

\section{Shoot Regeneration and Callus Induction}

In this study, there 4 types of explants (shoot, stem, root, and petiole) were tested in several combinations of 6-Benzylaminopurine (BAP) and Naphaleneacetic acid (NAA). The explants were cut into small fragments about $1.0 \mathrm{~cm}$ x 1.0 $\mathrm{cm}$ and were placed on the top of basic medium that contained desired combination of hormones. The explants were incubated in constant condition, 16 hours of artificial light and 8 hours of darkness at $23 \pm 3{ }^{\circ} \mathrm{C}$.

\section{Statistical Analysis}

The experiments were conducted in completely randomized design (CRD) and there were 10 replicates per treatment and each treatment was repeated in three sets. The data were analyzed by Analysis of Varience (ANOVA) followed by Duncan's multiple range test $p=0.5$. Data analyses were carried out by using SPSS version 23.

\section{RESUlTS AND Discussion}

\section{A. Effect of BAP and NAA on In vitro stem, leaf and root} explants

Effects of BAP and NAA on In vitro stem, leaf and root explants for number of shoot, number of root and callus height. For stem explants number of shoot only respond for Treatment $7(1.0 \mathrm{mg} / \mathrm{L}$ BAP and $0.5 \mathrm{mg} / \mathrm{L}$ NAA $)$ with $3.000 \pm 0.000$, at Treatment $11(1.0 \mathrm{mg} / \mathrm{L} \mathrm{BAP}$ and $1.0 \mathrm{mg} / \mathrm{L}$ NAA) with $6.600 \pm 5.963$, Treatment $8(2.0 \mathrm{mg} / \mathrm{L}$ BAP and 0.5 $\mathrm{mg} / \mathrm{L}$ NAA) with $1.000 \pm 1.000$ and the highest is treatment 14 $(0.5 \mathrm{mg} / \mathrm{L}$ BAP and $\mathrm{mg} / \mathrm{L}$ NAA) with $15.400 \pm 7.56$. But for another treatment there were no respond and effect of BAP and NAA for stem explants. For number of root for leaf explants only two treatments gave respond. There were Treatment 11(1.0 $\mathrm{mg} / \mathrm{L}$ BAP and $1.0 \mathrm{mg} / \mathrm{L}$ NAA) with $1.500 \pm 0.670$ and Treatment $16(2.0 \mathrm{mg} / \mathrm{L}$ BAP and $2.0 \mathrm{mg} / \mathrm{L}$ NAA $)$ with $0.900 \pm 0.498$. But for another treatment there were no respond. Lastly for root explants response for number of shoot. There were no respond. This was because for root explants, hairy root was observed for week 8 .

For number of roots for stem explants. All the treatment respond to the explants except treatment 1(basal medium). There were treatment $6(0.5 \mathrm{mg} / \mathrm{L} \mathrm{BAP}$ and $0.5 \mathrm{mg} / \mathrm{L} \mathrm{NAA})$ with $0.900 \pm 0.458$, Treatment $7(1.0 \mathrm{mg} / \mathrm{L}$ BAP and $0.5 \mathrm{mg} / \mathrm{L}$ NAA) with $0.900 \pm 0.640$, Treatment $8(2.0 \mathrm{mg} / \mathrm{L}$ BAP and 0.5 $\mathrm{mg} / \mathrm{L}$ NAA) with $0.600 \pm 0.600$, Treatment $10(0.5 \mathrm{mg} / \mathrm{L}$ BAP and $1.0 \mathrm{mg} / \mathrm{L}$ NAA) with $0.500 \pm 0.342$, Treatment $11(1.0 \mathrm{mg} / \mathrm{L}$ BAP and $1.0 \mathrm{mg} / \mathrm{L}$ NAA) with $0.600 \pm 0.400$, Treatment $12(2.0$ $\mathrm{mg} / \mathrm{L}$ BAP and $1.0 \mathrm{mg} / \mathrm{L}$ NAA) with $0.600 \pm 0.400$, Treatment $14(0.5 \mathrm{mg} / \mathrm{L}$ BAP and $2.0 \mathrm{mg} / \mathrm{L}$ NAA) with $1.600 \pm 0.792$, Treatment $15(1.0 \mathrm{mg} / \mathrm{L} \mathrm{BAP}$ and $2.0 \mathrm{mg} / \mathrm{L}$ NAA $)$ with $0.300 \pm 0.300$, Treatment $16(2.0 \mathrm{mg} / \mathrm{L} \mathrm{BAP}$ and $2.0 \mathrm{mg} / \mathrm{L} \mathrm{NAA})$ with $0.300 \pm 0.300$. Only Treatment 1 (MS basal medium) did not response. For the number of root for leaf explants. There were no respond because leaf explants only induced callus. For root the number of roots, the respond for Treatment 1 (MS basal medium) with $0.600 \pm 0.305$, Treatment $8(2.0 \mathrm{mg} / \mathrm{L}$ BAP and $0.5 \mathrm{mg} / \mathrm{L}$ NAA) with $0.600 \pm 0.600$, Treatment $12(2.0 \mathrm{mg} / \mathrm{L}$ BAP and $1.0 \mathrm{mg} / \mathrm{L}$ NAA) with $0.400 \pm 0.267$, Treatment 14 (0.5 $\mathrm{mg} / \mathrm{L}$ BAP and $2.0 \mathrm{mg} / \mathrm{L}$ NAA) with $0.600 \pm 0.600$, Treatment $15(1.0 \mathrm{mg} / \mathrm{L}$ BAP and $2.0 \mathrm{mg} / \mathrm{L}$ NAA) with $1.200 \pm 0.679$ and Treatment $16(2.0 \mathrm{mg} / \mathrm{L}$ BAP and $2.0 \mathrm{mg} / \mathrm{L}$ NAA $)$ with $0.700 \pm 0.700$. The other treatments did not respond.

Callus height for stem explants the best treatment was Treatment $6(0.5 \mathrm{mg} / \mathrm{L}$ BAP and $0.5 \mathrm{mg} / \mathrm{L}$ NAA $)$ with $0.980 \pm 0.231 \mathrm{~cm}$, Treatment $8(2.0 \mathrm{mg} / \mathrm{L}$ BAP and $0.5 \mathrm{mg} / \mathrm{L}$ NAA) with $0.302 \pm 0.106 \mathrm{~cm}$, Treatment $11(1.0 \mathrm{mg} / \mathrm{L}$ BAP and $1.0 \mathrm{mg} / \mathrm{L}$ NAA) with $0.860 \pm 1.91 \mathrm{~cm}$, Treatment $14(0.5 \mathrm{mg} / \mathrm{L}$ BAP and $2.0 \mathrm{mg} / \mathrm{L}$ NAA) with $0.880 \pm 0.377 \mathrm{~cm}$, Treatment 15 $(2.0 \mathrm{mg} / \mathrm{L}$ BAP and $2.0 \mathrm{mg} / \mathrm{L}$ NAA) with $0.120 \pm 0.068 \mathrm{~cm}$ and lastly Treatment $16(2.0 \mathrm{mg} / \mathrm{L} \mathrm{BAP}$ and $2.0 \mathrm{mg} / \mathrm{L} \mathrm{NAA})$ with $0 . .150 \pm 0.077 \mathrm{~cm}$. The another treatments did not respond to the stem explants. For the leaf explants the highest respond is for Treatment $11(1.0 \mathrm{mg} / \mathrm{L}$ BAP and $1.0 \mathrm{mg} / \mathrm{L} \mathrm{NAA})$ is $1.220 \pm 0.153 \mathrm{~cm}$, Treatment $16(2.0 \mathrm{mg} / \mathrm{L}$ BAP and $2.0 \mathrm{mg} / \mathrm{L}$ NAA) with $0.520 \pm 0.152 \mathrm{~cm}$, Treatment $7(0.5 \mathrm{mg} / \mathrm{L}$ BAP and $0.5 \mathrm{mg} / \mathrm{L}$ NAA) with $0.320 \pm 0.179 \mathrm{~cm}$, Treatment $14(0.5 \mathrm{mg} / \mathrm{L}$ BAP and $2.0 \mathrm{mg} / \mathrm{L}$ NAA) with $0.250 \pm 0.079 \mathrm{~cm}$ Treatment 10 $(0.5 \mathrm{mg} / \mathrm{L}$ BAP and 1.0 .

TABLE I: EFFECT OF BAP AND NAA ON IN VITRO FOR NUMBER OF SHOOTS OF STEMS, LEAF AND ROOT EXPLANTS

\begin{tabular}{|c|c|c|c|}
\hline \multirow[t]{2}{*}{ TREATMENT } & \multicolumn{3}{|c|}{ NO. OF SHOOT } \\
\hline & STEM & LEAF & ROOT \\
\hline $\begin{array}{l}0.0 \mathrm{mg} / \mathrm{L} \text { BAP }+0.0 \\
\mathrm{mg} / \mathrm{L} \text { NAA }\end{array}$ & - & - & - \\
\hline $\begin{array}{l}0.5 \mathrm{mg} / \mathrm{L} \text { BAP }+0.5 \\
\mathrm{mg} / \mathrm{L} \text { NAA }\end{array}$ & - & - & - \\
\hline $\begin{array}{l}1.0 \mathrm{mg} / \mathrm{L} \text { BAP }+0.5 \\
\mathrm{mg} / \mathrm{L} \text { NAA }\end{array}$ & $3.000 \pm 0.000$ & - & - \\
\hline $\begin{array}{l}2.0 \mathrm{mg} / \mathrm{L} \text { BAP }+0.5 \\
\mathrm{mg} / \mathrm{L} \text { NAA }\end{array}$ & $1.000 \pm 1.000$ & - & - \\
\hline $\begin{array}{l}0.5 \mathrm{mg} / \mathrm{L} \text { BAP }+1.0 \\
\mathrm{mg} / \mathrm{L} \text { NAA }\end{array}$ & - & - & - \\
\hline $\begin{array}{l}1.0 \mathrm{mg} / \mathrm{L} \mathrm{BAP}+1.0 \\
\mathrm{mg} / \mathrm{L} \text { NAA }\end{array}$ & $6.600 \pm 5.963$ & $1.500 \pm 0.671$ & - \\
\hline $\begin{array}{l}2.0 \mathrm{mg} / \mathrm{L} \mathrm{BAP}+1,0 \\
\mathrm{mg} / \mathrm{L} \text { NAA }\end{array}$ & - & - & - \\
\hline $\begin{array}{l}0.5 \mathrm{mg} / \mathrm{L} \text { BAP }+2.0 \\
\mathrm{mg} / \mathrm{L} \text { NAA }\end{array}$ & $15.400 \pm 7.560$ & - & - \\
\hline $\begin{array}{l}1.0 \mathrm{mg} / \mathrm{L} \text { BAP }+2.0 \\
\mathrm{mg} / \mathrm{L} \text { NAA }\end{array}$ & - & - & - \\
\hline $\begin{array}{l}2.0 \mathrm{mg} / \mathrm{L} \mathrm{BAP}+2.0 \\
\mathrm{mg} / \mathrm{L} \mathrm{NAA}\end{array}$ & - & $0.900 \pm 0.498$ & - \\
\hline
\end{tabular}

Mean \pm SE, $n=30$. Mean is different significantly at $p=0.5$

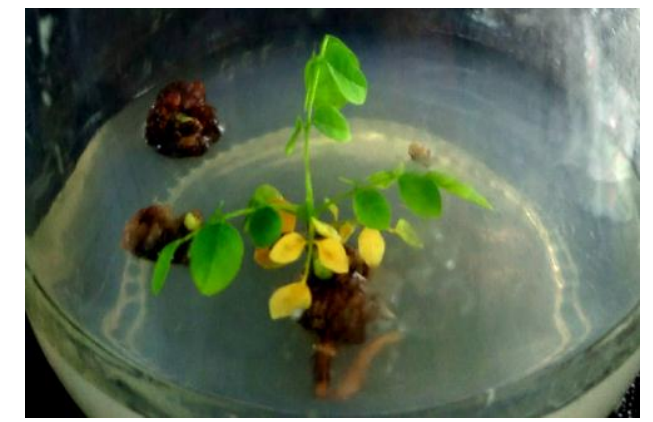

Fig. 1 shows shoot regeneration on (a) stem explants cultured on 0.5 $\mathrm{mg} / \mathrm{L} \mathrm{BAP}+2.0 \mathrm{mg} / \mathrm{L}$ NAA for 8 week 
TABLE II: EFFECT OF BAP AND NAA ON IN VITRO FOR NUMBER OF ROOT OF STEM, LEAF AND ROOT EXPLANTS.

\begin{tabular}{|c|c|c|c|}
\hline \multirow[t]{2}{*}{ TREATMENT } & \multicolumn{3}{|c|}{ NO. OF ROOT } \\
\hline & STEM & LEAF & ROOT \\
\hline $\begin{array}{l}0.0 \mathrm{mg} / \mathrm{L} \text { BAP }+ \\
0.0 \mathrm{mg} / \mathrm{L} \mathrm{NAA}\end{array}$ & - & - & $0.600 \pm 0.305$ \\
\hline $\begin{array}{l}0.0 \mathrm{mg} / \mathrm{L} \text { BAP }+ \\
0.5 \mathrm{mg} / \mathrm{L} \text { NAA }\end{array}$ & $0.900 \pm 0.458$ & - & - \\
\hline $\begin{array}{l}0.5 \mathrm{mg} / \mathrm{L} \mathrm{BAP}+ \\
0.5 \mathrm{mg} / \mathrm{L} \text { NAA }\end{array}$ & $0.900 \pm 0.640$ & - & - \\
\hline $\begin{array}{l}1.0 \mathrm{mg} / \mathrm{L} \mathrm{BAP} \mathrm{+} \\
0.5 \mathrm{mg} / \mathrm{L} \text { NAA }\end{array}$ & $0.600 \pm 0.600$ & - & $0.600 \pm 0.600$ \\
\hline $\begin{array}{l}1.5 \mathrm{mg} / \mathrm{L} \mathrm{BAP}+ \\
0.5 \mathrm{mg} / \mathrm{L} \text { NAA }\end{array}$ & $0.500 \pm 0.342$ & - & - \\
\hline $\begin{array}{l}0.5 \mathrm{mg} / \mathrm{L} \mathrm{BAP}+ \\
1.0 \mathrm{mg} / \mathrm{L} \mathrm{NAA}\end{array}$ & $0.600 \pm 0.400$ & - & - \\
\hline $\begin{array}{l}1.0 \mathrm{mg} / \mathrm{L} \mathrm{BAP} \mathrm{+} \\
1.0 \mathrm{mg} / \mathrm{L} \mathrm{NAA}\end{array}$ & $0.600 \pm 0.400$ & - & $0.400 \pm 0.267$ \\
\hline $\begin{array}{l}1.5 \mathrm{mg} / \mathrm{L} \mathrm{BAP}+ \\
1.0 \mathrm{mg} / \mathrm{L} \mathrm{NAA}\end{array}$ & $1.600 \pm 0.792$ & - & $0.600 \pm 0.600$ \\
\hline $\begin{array}{l}0.0 \mathrm{mg} / \mathrm{L} \mathrm{BAP}+ \\
0.0 \mathrm{mg} / \mathrm{L} \mathrm{NAA}\end{array}$ & $0.300 \pm 0.300$ & - & $1.200 \pm 0.679$ \\
\hline $\begin{array}{l}0.0 \mathrm{mg} / \mathrm{L} \text { BAP + } \\
0.5 \mathrm{mg} / \mathrm{L} \text { NAA }\end{array}$ & $0.300 \pm 0.300$ & - & $0.700 \pm 0.700$ \\
\hline
\end{tabular}

Mean \pm SE, $n=30$. Mean is different significantly at $p=0.5$

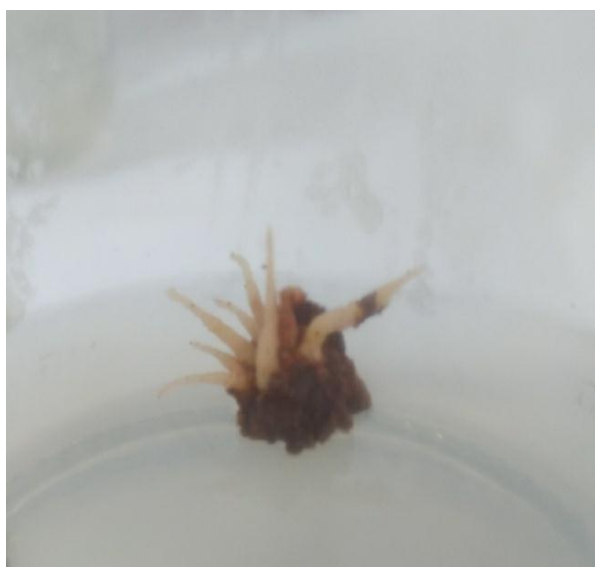

. Fig. 2 shows root regeneration on (a) root explant cultured on 1.5 $\mathrm{mg} / \mathrm{L} \mathrm{BAP}+1.0 \mathrm{mg} / \mathrm{L} \mathrm{NAA}$ for 8 week
TABLE III: EFFECT OF BAP AND NAA ON IN VITRO FOR CALLUS HEIGHT OF STEMS, LEAF AND ROOT EXPLANTS.

\begin{tabular}{|c|c|c|c|}
\hline \multirow[t]{2}{*}{ TREATMENT } & \multicolumn{3}{|c|}{$\begin{array}{l}\text { CALLUS THEIGHT (CM) } \\
\text { (CM }\end{array}$} \\
\hline & STEM & LEAF & ROOT \\
\hline $\begin{array}{l}0.0 \mathrm{mg} / \mathrm{L} \text { BAP } \\
+0.0 \mathrm{mg} / \mathrm{L} \\
\text { NAA }\end{array}$ & 0 & 0 & $0.140 \pm 0.099$ \\
\hline $\begin{array}{l}0.5 \mathrm{mg} / \mathrm{L} \text { BAP } \\
+0.5 \mathrm{mg} / \mathrm{L} \\
\text { NAA }\end{array}$ & $0.980 \pm 0.231$ & $0.380 \pm 0.118$ & $0.200 \pm 0.133$ \\
\hline $\begin{array}{l}1.0 \mathrm{mg} / \mathrm{L} \text { BAP } \\
+0.5 \mathrm{mg} / \mathrm{L} \\
\text { NAA }\end{array}$ & 0 & $0.320 \pm 0.179$ & $0.200 \pm 0.158$ \\
\hline $\begin{array}{l}2.0 \mathrm{mg} / \mathrm{L} \text { BAP } \\
+0.5 \mathrm{mg} / \mathrm{L} \\
\text { NAA }\end{array}$ & $0.302 \pm 0.106$ & 0 & $0.060 \pm 0.060$ \\
\hline $\begin{array}{l}0.5 \mathrm{mg} / \mathrm{L} \mathrm{BAP} \\
+1.0 \mathrm{mg} / \mathrm{L} \\
\mathrm{NAA}\end{array}$ & 0 & $0.300 \pm 0.300$ & $0.220 \pm 0.105$ \\
\hline $\begin{array}{l}1.0 \mathrm{mg} / \mathrm{L} \text { BAP } \\
+1.0 \mathrm{mg} / \mathrm{L} \\
\text { NAA }\end{array}$ & $0.860 \pm 0.191$ & $1.220 \pm 0.153$ & $0.300 \pm 0.300$ \\
\hline $\begin{array}{l}2.0 \mathrm{mg} / \mathrm{L} \text { BAP } \\
+1,0 \mathrm{mg} / \mathrm{L} \\
\text { NAA }\end{array}$ & 0 & 0 & 0 \\
\hline $\begin{array}{l}0.5 \mathrm{mg} / \mathrm{L} \text { BAP } \\
+2.0 \mathrm{mg} / \mathrm{L} \\
\text { NAA }\end{array}$ & $0.880 \pm 0.377$ & $0.250 \pm 0.079$ & $0.120 \pm 0.085$ \\
\hline $\begin{array}{l}1.0 \mathrm{mg} / \mathrm{L} \mathrm{BAP} \\
+2.0 \mathrm{mg} / \mathrm{L} \\
\mathrm{NAA}\end{array}$ & $0.120 \pm 0.068$ & 0 & 0 \\
\hline $\begin{array}{l}2.0 \mathrm{mg} / \mathrm{L} \text { BAP } \\
+2.0 \mathrm{mg} / \mathrm{L} \\
\text { NAA }\end{array}$ & $0.150 \pm 0.077$ & $0.520 \pm 0.152$ & $0.100 \pm 0.100$ \\
\hline
\end{tabular}

Mean $\pm S E, n=30$. Mean is different significantly at $p=0.5$

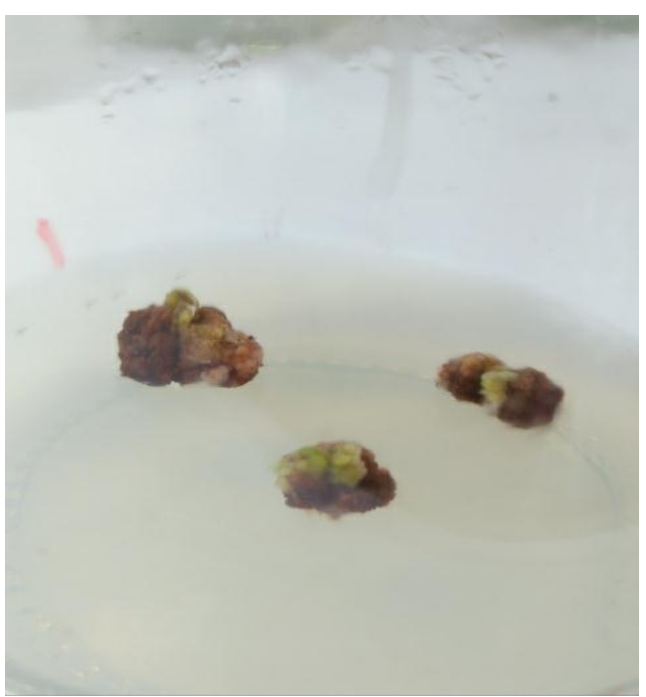

Fig. 3 shows root regeneration on (a) leaf explant cultured on $1.5 \mathrm{mg} / \mathrm{L}$ $\mathrm{BAP}+1.0 \mathrm{mg} / \mathrm{L}$ NAA for 8 week

B. Combinations and concentrations of plant growth regulators (BAP and NAA) gave response to callus induction on Averhoa carambola

From this experiment, the various combinations and concentrations of plant growth regulators (BAP and NAA) gave response to callus induction on Averhoa carambola leaf explants, the observation is recorded at 4 and 8 weeks-old. Performance of callus induction was inspected in two aspects, callus percentage $(\%)$ and average weight of callus. During the week 4 of the culture, Treatment $6(0.5 \mathrm{mg} / \mathrm{L} \mathrm{BAP}$ and $0.5 \mathrm{mg} / \mathrm{L}$ 
NAA), Treatment $8(2.0 \mathrm{mg} / \mathrm{L}$ BAP and $0.5 \mathrm{mg} / \mathrm{L}$ NAA) ,Treatment $11(1.0 \mathrm{mg} / \mathrm{L}$ BAP and $1.0 \mathrm{mg} / \mathrm{L} \mathrm{NAA})$ and Treatment $16(2.0 \mathrm{mg} / \mathrm{L}$ BAP and $2.0 \mathrm{mg} / \mathrm{L}$ NAA showed best response in callus induction, they gave $100 \%$ callus induction and medium size callus. At week 8, Treatment $6(0.5 \mathrm{mg} / \mathrm{L} \mathrm{BAP}$ and $0.5 \mathrm{mg} / \mathrm{L}$ NAA) Treatment $8(2.0 \mathrm{mg} / \mathrm{L} \mathrm{BAP}$ and $0.5 \mathrm{mg} / \mathrm{L}$ NAA), Treatment $11(1.0 \mathrm{mg} / \mathrm{L}$ BAP and $1.0 \mathrm{mg} / \mathrm{L} \mathrm{NAA})$ and Treatment $16(2.0 \mathrm{mg} / \mathrm{L}$ BAP and $2.0 \mathrm{mg} / \mathrm{L}$ NAA $)$ were among the combination and concentration that perform the best. All the treatment have $100 \%$ of callus. But the highest average weight is treatment $10(0.5 \mathrm{mg} / \mathrm{L} \mathrm{BAP}$ and $1.0 \mathrm{mg} / \mathrm{L} \mathrm{NAA})$ with $0.404 \pm 0.041 \mathrm{~g}$.

TABLE 4 : EFFECT OF BAP AND NAA ON IN VITRO CALLUS INDUCTION ON LEAF EXPLANTS OF AVERHOA CARAMBOLA

\begin{tabular}{|c|c|c|}
\hline Treatment & $\begin{array}{l}\text { Average weight of callus } \\
\text { at week } 8\end{array}$ & Callus condition \\
\hline $\begin{array}{l}0.0 \mathrm{mg} / \mathrm{L} \mathrm{BAP}+0.0 \\
\mathrm{mg} / \mathrm{L} \text { NAA }\end{array}$ & $0.020 \pm 0.002$ & Brown \\
\hline $\begin{array}{l}0.5 \mathrm{mg} / \mathrm{L} \text { BAP }+0.5 \\
\mathrm{mg} / \mathrm{L} \text { NAA }\end{array}$ & $0.151 \pm 0.052$ & Brown \\
\hline $\begin{array}{l}1.0 \mathrm{mg} / \mathrm{L} \text { BAP }+0.5 \\
\mathrm{mg} / \mathrm{L} \text { NAA }\end{array}$ & $0.193 \pm 0.043$ & Brown \\
\hline $\begin{array}{l}2.0 \mathrm{mg} / \mathrm{L} \text { BAP }+0.5 \\
\mathrm{mg} / \mathrm{L} \text { NAA }\end{array}$ & $0.065 \pm 0.022$ & Brown \\
\hline $\begin{array}{l}0.5 \mathrm{mg} / \mathrm{L} \mathrm{BAP}+1.0 \\
\mathrm{mg} / \mathrm{L} \text { NAA }\end{array}$ & $0.404 \pm 0.042$ & Brown \\
\hline $\begin{array}{l}1.0 \mathrm{mg} / \mathrm{L} \mathrm{BAP}+1.0 \\
\mathrm{mg} / \mathrm{L} \text { NAA }\end{array}$ & $0.197 \pm 0.047$ & Brown \\
\hline $\begin{array}{l}2.0 \mathrm{mg} / \mathrm{L} \text { BAP }+1.0 \\
\mathrm{mg} / \mathrm{L} \text { NAA }\end{array}$ & $0.039 \pm 0.017$ & Brown \\
\hline $\begin{array}{l}0.5 \mathrm{mg} / \mathrm{L} \text { BAP }+2.0 \\
\mathrm{mg} / \mathrm{L} \text { NAA }\end{array}$ & $0.222 \pm 0.049$ & Brown \\
\hline $\begin{array}{l}1.0 \mathrm{mg} / \mathrm{L} \mathrm{BAP}+2.0 \\
\mathrm{mg} / \mathrm{L} \text { NAA }\end{array}$ & $0.010 \pm 0.000$ & Brown \\
\hline $\begin{array}{l}2 . .0 \mathrm{mg} / \mathrm{L} \mathrm{BAP}+2.0 \\
\mathrm{mg} / \mathrm{L} \text { NAA }\end{array}$ & $0.164 \pm 0.072$ & Brown \\
\hline
\end{tabular}

\section{Stem Explants}

Same experiment was conducted toward stem explants of Averhoa carambola. During the week 4 of the culture, only Treatment $6(0.5 \mathrm{mg} / \mathrm{L} \mathrm{BAP}$ and $0.5 \mathrm{mg} / \mathrm{L} \mathrm{NAA})$, perform the best which gave $100 \%$ callus induction and medium size callus. This is because for Averhoa carambola stem explants is the best part of plants to growth. At week 8, callus in Treatment $6(0.5$ $\mathrm{mg} / \mathrm{L} \mathrm{BAP}$ and $0.5 \mathrm{mg} / \mathrm{L} \mathrm{NAA}$ ) continued to perform and give a large size callus. However, the heaviest callus for petiole explants was obtained from Treatment 15 with average weight $0.130 \pm 0.002 \mathrm{~g}$. This observation might due to the callus from Treatment 10 grow long healthy roots, while the other treatment the root growth not very well.
TABLE 5: EFFECT OF BAP AND NAA ON IN VITRO CALLUS INDUCTION ON STEM EXPLANTS OF AVERHOA CARAMBOLA

\begin{tabular}{|c|c|c|}
\hline Treatment & $\begin{array}{l}\text { Average weight of callus at } \\
\text { week } 8\end{array}$ & $\begin{array}{l}\text { Callus } \\
\text { condition }\end{array}$ \\
\hline $\begin{array}{l}0.0 \mathrm{mg} / \mathrm{L} \text { BAP } \\
+0.0 \mathrm{mg} / \mathrm{L} \\
\text { NAA }\end{array}$ & $0.019 \pm 0.004$ & Brown \\
\hline $\begin{array}{l}0.5 \mathrm{mg} / \mathrm{L} \text { BAP } \\
+0.5 \mathrm{mg} / \mathrm{L} \\
\mathrm{NAA}\end{array}$ & $0.045 \pm 0.028$ & Brown \\
\hline $\begin{array}{l}1.0 \mathrm{mg} / \mathrm{L} \text { BAP } \\
+0.5 \mathrm{mg} / \mathrm{L} \\
\mathrm{NAA}\end{array}$ & $0.041 \pm 0.025$ & Brown \\
\hline $\begin{array}{l}2.0 \mathrm{mg} / \mathrm{L} \text { BAP } \\
+0.5 \mathrm{mg} / \mathrm{L} \\
\mathrm{NAA}\end{array}$ & $0.029 \pm 0.005$ & Brown \\
\hline $\begin{array}{l}0.5 \mathrm{mg} / \mathrm{L} \mathrm{BAP} \\
+1.0 \mathrm{mg} / \mathrm{L} \\
\text { NAA }\end{array}$ & $0.018 \pm 0.003$ & Brown \\
\hline $\begin{array}{l}1.0 \mathrm{mg} / \mathrm{L} \text { BAP } \\
+1.0 \mathrm{mg} / \mathrm{L} \\
\text { NAA }\end{array}$ & $0.042 \pm 0.015$ & Brown \\
\hline $\begin{array}{l}2.0 \mathrm{mg} / \mathrm{L} \text { BAP } \\
+1.0 \mathrm{mg} / \mathrm{L} \\
\text { NAA }\end{array}$ & $0.037 \pm 0.008$ & Brown \\
\hline $\begin{array}{l}0.5 \mathrm{mg} / \mathrm{L} \mathrm{BAP} \\
+2.0 \mathrm{mg} / \mathrm{L} \\
\mathrm{NAA}\end{array}$ & $0.037 \pm 0.009$ & Brown \\
\hline $\begin{array}{l}1.0 \mathrm{mg} / \mathrm{L} \mathrm{BAP} \\
+2.0 \mathrm{mg} / \mathrm{L} \\
\mathrm{NAA}\end{array}$ & $0.118 \pm 0.025$ & Brown \\
\hline $\begin{array}{l}2 . .0 \mathrm{mg} / \mathrm{L} \text { BAP } \\
+2.0 \mathrm{gg} / \mathrm{L} \\
\mathrm{NAA}\end{array}$ & $0.019 \pm 0.003$ & Brown \\
\hline
\end{tabular}

\section{Root Explants}

Effect of various combinations and concentrations of BAP and NAA was also studied on root explants of Averhoa carambola. During the week 4 of the culture, Treatment $6(0.5$ $\mathrm{mg} / \mathrm{L} \mathrm{BAP}$ and $0.5 \mathrm{mg} / \mathrm{L}$ NAA) showed best and the higher percentage of callus formation. the heaviest callus for root explants was obtained from Treatment $15(1.0 \mathrm{mg} / \mathrm{L}$ BAP and $2.0 \mathrm{mg} / \mathrm{L}$ NAA) with average weight $0.118 \pm 0.025 \mathrm{~g}$. However Treatment 16 was the best treatment for root explants because formation of root growth at treatment 16 . 
TABLE 6: EFFECT OF BAP AND NAA ON IN VITRO CALLUS INDUCTION ON ROOT EXPLANTS OF AVERHOA CARAMBOLA

\begin{tabular}{|c|c|c|}
\hline Treatment & $\begin{array}{l}\text { Average weight of callus at } \\
\text { week } 8\end{array}$ & Callus condition \\
\hline $\begin{array}{l}0.0 \mathrm{mg} / \mathrm{L} \mathrm{BAP} \\
+0.0 \mathrm{mg} / \mathrm{L} \\
\text { NAA }\end{array}$ & $0.037 \pm 0.003$ & Brown \\
\hline $\begin{array}{l}0.5 \mathrm{mg} / \mathrm{L} \mathrm{BAP} \\
+0.5 \mathrm{mg} / \mathrm{L} \\
\mathrm{NAA}\end{array}$ & $0.279 \pm 0.070$ & Brown \\
\hline $\begin{array}{l}1.0 \mathrm{mg} / \mathrm{L} \mathrm{BAP} \\
+0.5 \mathrm{mg} / \mathrm{L} \\
\mathrm{NAA}\end{array}$ & $0.034 \pm 0.007$ & Brown \\
\hline $\begin{array}{l}2.0 \mathrm{mg} / \mathrm{L} \mathrm{BAP} \\
+0.5 \mathrm{mg} / \mathrm{L} \\
\text { NAA }\end{array}$ & $0.033 \pm 0.008$ & Brown \\
\hline $\begin{array}{l}0.5 \mathrm{mg} / \mathrm{L} \mathrm{BAP} \\
+1.0 \mathrm{mg} / \mathrm{L} \\
\mathrm{NAA}\end{array}$ & $0.040 \pm 0.016$ & Brown \\
\hline $\begin{array}{l}1.0 \mathrm{mg} / \mathrm{L} \mathrm{BAP} \\
+1.0 \mathrm{mg} / \mathrm{L} \\
\text { NAA }\end{array}$ & $0.032 \pm 0.006$ & Brown \\
\hline $\begin{array}{l}2.0 \mathrm{mg} / \mathrm{L} \mathrm{BAP} \\
+1.0 \mathrm{mg} / \mathrm{L} \\
\text { NAA }\end{array}$ & $0.024 \pm 0.004$ & Brown \\
\hline $\begin{array}{l}0.5 \mathrm{mg} / \mathrm{L} \mathrm{BAP} \\
+2.0 \mathrm{mg} / \mathrm{L} \\
\mathrm{NAA}\end{array}$ & $0.043 \pm 0.007$ & Brown \\
\hline $\begin{array}{l}1.0 \mathrm{mg} / \mathrm{L} \mathrm{BAP} \\
+2.0 \mathrm{mg} / \mathrm{L} \\
\mathrm{NAA}\end{array}$ & $0.013 \pm 0.002$ & Brown \\
\hline $\begin{array}{l}2 . .0 \mathrm{mg} / \mathrm{L} \\
\mathrm{BAP}+2.0 \\
\mathrm{mg} / \mathrm{L} \text { NAA }\end{array}$ & $0.010 \pm 0.000$ & Brown \\
\hline
\end{tabular}

Mean \pm SE, $n=30$. Mean is different significantly at $p=0.5$

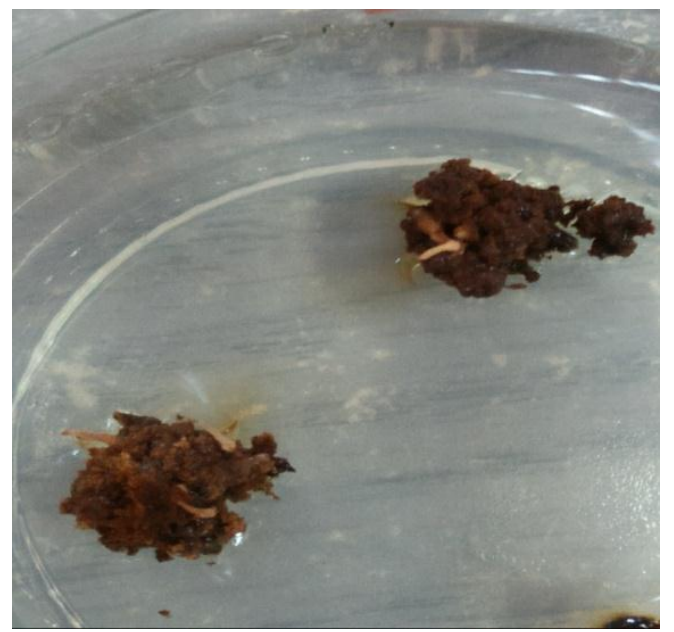

Fig. 4 Combinations and concentrations of plant growth regulators (BAP and NAA) gave response to callus induction on Averhoa carambol

\section{CONCLUSION}

In conclusion, in vitro regeneration of Averhoa carambola had been successfully obtained from explants culture on various combination and concentration of BAP and NAA. Two samples, one stem explant in BAP $0.5 \mathrm{mg} / \mathrm{L}$ and NAA $2.0 \mathrm{mg} / \mathrm{L}$ and one shoot explant in BAP $1.0 \mathrm{mg} / \mathrm{L}$ and NAA $2.0 \mathrm{mg} / \mathrm{L}$ successfully developed into complete plants.

For callus induction, it was observed that leaf explant produced the optimum callus when cultured on BAP $0.5 \mathrm{mg} / \mathrm{L}$ and NAA $0.5 \mathrm{mg} / \mathrm{L}$ medium. Meanwhile, shoot regeneration frequency was relatively low in this experiment. Healthy shoots developed in a stem explants in BAP $0.5 \mathrm{mg} / \mathrm{L}$ and NAA 2.0 $\mathrm{mg} / \mathrm{L}$ and a shoot explants in BAP $1.0 \mathrm{mg} / \mathrm{L}$ and NAA $1.0 \mathrm{mg} / \mathrm{L}$. Other the others hand, root formation was more prevalent in this experiment. Leaf explants cultured on BAP $1.0 \mathrm{mg} / \mathrm{L}$ and NAA $0.5 \mathrm{mg} / \mathrm{L}$ and stem explants in BAP $2.0 \mathrm{mg} / \mathrm{L}$ and NAA 0.5 $\mathrm{mg} / \mathrm{L}$ (Figure 4.4) performed the best in forming functional roots. In the present study, it showed that in vitro regeneration of Averhoa carambola were influenced by two factors such as type of explants and different concentration and combination plant growth regulators used. Besides, other factors not under this study such as types of plant growth regulators [9] and [5] sources; age, size, and overall quality of the plant [6] will also affect the in vitro regeneration of explants.

\section{REFERENCES}

[1] Ahmed, S., and Beigh, S.H. (2009) Ascorbic acid,carotenoids, total pthenolic content and antioxidant activity of various genotypes of Brassica Oleeacea encephala. J. Med. Biol. Sci., 3, pp. 1-8.

[2] Arnous, A., Makris, D.P., and Kefalas, P. (2001) Effect ofprincipal polypthenolic components in relation to antioxidant characteristics of aged red wines. J. Agric Food Cthem., 49(12), pp. 5736-5742. https://doi.org/10.1021/jf010827s

[3] Arockiasamy, Prakash and Ignachimutu. (2002). Direct organogenesis from mature leaf and petiole explants of Eryngium foetidum L. Biological planetarium 45(1) : 129-132.

[4] Bethera, P.R., Nayak, P., Thirunavoukkarasu, M. And Sahoo, S.: Indian J. Plant Physiol,13(3): 258-265 (2008).

[5] Bhojwani and Razdan. (1996). Plant tissue culture: theory and practice. Elsevier, Amsterdam.

[6] Dodds, J. H., L. Roberts, W. (1995). Experiments in Plant Tissue Culture. USA:Cambridge University Press,

[7] Donnarumma, F., Capuana, M., Vettori, C., Giannini, R., Indorate, C., and Matromei,

[8] G.(2011). Isolation and characterization of bacterial colonies from seeds and invitro cultures of Fraxinus spp. From Italian sites. Plant Biology, 13(1), 169-176.

[9] Friml, J. (2003). Auxin transport - shaping the plant. In: Curr. Opin. Plant. Biol. 6, 1.6.

[10] Gamborg, O.L. dan Philips, G. C (pnyt.) 1995. Plant Cell, Tissue and Organ Culture. Springer, Berlin 